\title{
Efficient photon detection from color centers in a diamond optical waveguide
}

\author{
D. Le Sage, ${ }^{1,2}$ L. M. Pham, ${ }^{3}$ N. Bar-Gill,,${ }^{1,2}$ C. Belthangady,,${ }^{1,2}$ M. D. Lukin, ${ }^{2}$ A. Yacoby, ${ }^{2}$ and R. L. Walsworth ${ }^{1,2, *}$ \\ ${ }^{1}$ Harvard-Smithsonian Center for Astrophysics, Cambridge, Massachusetts 02138, USA \\ ${ }^{2}$ Physics Department, Harvard University, Cambridge, Massachusetts 02138, USA \\ ${ }^{3}$ School of Engineering and Applied Sciences, Harvard University, Cambridge, Massachusetts 02138, USA
}

(Received 24 December 2011; published 23 March 2012)

\begin{abstract}
A common limitation of experiments using color centers in diamond is the poor photon collection efficiency of microscope objectives due to refraction at the diamond interface. We present a simple and effective technique to detect a large fraction of photons emitted by color centers within a planar diamond sample by detecting light that is guided to the edges of the diamond via total internal reflection. We describe a prototype device using this "side-collection" technique, which provides a photon collection efficiency $\approx 47 \%$ and a photon detection efficiency $\approx 39 \%$. We apply the enhanced signal-to-noise ratio gained from side collection to ac magnetometry using ensembles of nitrogen-vacancy (NV) color centers, and demonstrate an ac magnetic field sensitivity $\approx 100 \mathrm{pT} / \sqrt{\mathrm{Hz}}$, limited by added noise in the prototype side-collection device. Technical optimization should allow significant further improvements in photon collection and detection efficiency as well as subpicotesla NV-diamond magnetic field sensitivity using the side-collection technique.
\end{abstract}

DOI: 10.1103/PhysRevB.85.121202

PACS number(s): 76.30.Mi, 42.79.Gn, 07.55.Ge, 78.55.-m

A wide variety of fluorescent point defect centers in diamond have been identified, with possible applications toward quantum information and biological imaging. ${ }^{1}$ One such color center that has attracted considerable attention is the negatively charged nitrogen-vacancy center (NV) [Fig. 1(a)], which has an electronic structure that allows for optical initialization and detection of the electronic spin, and coherent manipulation of the spin state, ${ }^{2}$ with long room-temperature spin coherence times. ${ }^{3}$ Demonstrated applications of NVs include single photon generation, ${ }^{4,5}$ quantum information processing, ${ }^{6-8}$ superresolution microscopy, ${ }^{9,10}$ nanoscale magnetometry using a single $\mathrm{NV},{ }^{11,12}$ and vector magnetic field imaging using ensembles of NVs. ${ }^{13}$ For all of these applications, the photon collection efficiency is critically important.

Conventionally, photons emitted from NVs in a bulk diamond substrate are collected using a microscope objective with a large numerical aperture (NA). However, refraction at the flat diamond interface reduces the effective NA of the objective by a factor equal to the refractive index of diamond $\left(n_{d}=2.4\right)$, resulting in a photon collection efficiency $\left(\eta_{c}\right)$ $\leqslant 10 \%$ (assuming a maximum objective NA of 1.49 ). ${ }^{14,15}$ Photon detection efficiencies $\left(\eta_{d}\right)<2 \%$ are typical when detector coupling and quantum efficiencies are taken into account.

We note that NVs in diamond nanostructures can avoid the $\eta_{c}$ degradation caused by a bulk diamond interface. For example, NVs in subwavelength-size diamond nanocrystals can be approximated as point emitters in the medium surrounding the nanocrystal, yielding higher $\eta_{c}$ but lower emission rates due to increased excited-state lifetime. ${ }^{4}$ Light from NVs inside fabricated diamond nanowires is primarily emitted along the nanowire symmetry axis, allowing $\eta_{c} \approx 40 \% .{ }^{5}$ However, NVs in diamond nanostructures typically have much shorter spin coherence times ${ }^{16}$ than those in bulk diamond. ${ }^{3,17}$ One approach to increase $\eta_{c}$ for NVs in bulk diamond is to construct a solid-immersion lens (SIL) out of the diamond substrate, using either macroscopic ${ }^{18}$ or microscopic ${ }^{19}$ fabrication techniques. Light from NVs at the center of a hemispherical diamond
SIL passes through the surface at normal incidence, allowing $20 \%<\eta_{c}<30 \%$. However, this improved photon collection efficiency is limited to NVs lying $<30 \mu \mathrm{m}$ from the center of a macroscopic SIL. ${ }^{18}$ For a larger field of view, or for large-volume ensemble measurements in bulk diamond, a different collection-enhancement technique is required.

Here we demonstrate an efficient fluorescence detection technique that uses the planar diamond chip containing the color centers as an optical waveguide. For this "sidecollection" technique, photons emitted by the color centers are confined between the two parallel diamond chip surfaces by total internal reflection (TIR) and guided to the sides (edges) of the diamond chip, where they pass through a filter onto a detector [Figs. 1(b) and 1(c)]. This technique avoids complicated fabrication procedures and allows efficient photon detection from color centers located anywhere within the diamond volume.

We estimated the expected efficiency of the side-collection technique using theoretical models of the average $\mathrm{NV}$ emission pattern and realistic approximations of the diamond chip geometry and acceptance angles of the detectors. ${ }^{14,15}$ We find that $\approx 91 \%$ of the NV fluorescence is confined by TIR between the polished (100) planar surfaces of the diamond chip, and $\approx 29 \%$ reaches the detectors on first incidence of the photons with the diamond chip sides (edges). Much of the light also undergoes TIR off the sides, but may reach the detectors after many reflections within the diamond. We therefore expect $29 \%<\eta_{c}<91 \%$ for the side-collection technique, depending on details of the experimental geometry, diamond chip surface properties, etc. Note that this estimate neglects reabsorption of NV fluorescence by other NVs in the diamond, which is a negligible effect for the diamond sample used in the measurements described below, but may be significant for diamonds with much larger NV concentrations. ${ }^{20}$

We constructed a prototype device to compare the sidecollection technique to fluorescence detection using a microscope objective [Figs. 2(a)-2(c)]. This prototype instrument employed a [100]-oriented diamond chip (4.3 $\mathrm{mm} \times$ 
(a)

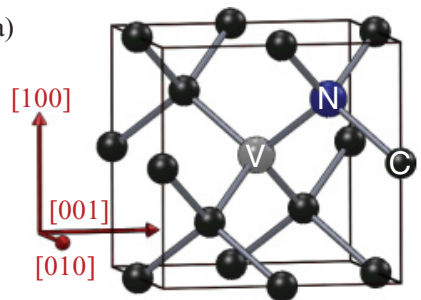

(c)

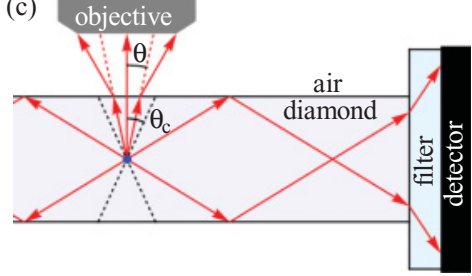

(b)

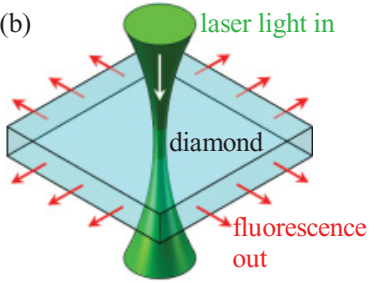

(d)

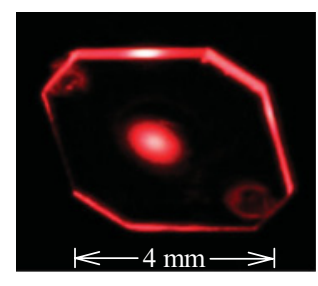

FIG. 1. (Color online) (a) The NV color center consists of a substitutional nitrogen atom $(\mathrm{N})$ adjacent to a vacancy $(\mathrm{V})$ in the diamond lattice. (b) In the side-collection technique, a focused laser beam excites color centers in a specific volume within the diamond, and much of the resulting fluorescence is detected after it exits one or more sides of the diamond waveguide. In the demonstration experiments reported here, four photodetectors are arranged around the four primary sides of the diamond chip. (c) Ray diagrams illustrate refraction at the diamond surface and light guided by total internal reflection above the critical angle $\left(\theta_{c}=24.6^{\circ}\right)$. (d) Red-filtered photograph of NV fluorescence from the diamond chip used in the demonstrations reported here. Guiding of NV fluorescence light is evident as a bright glow around the diamond's perimeter, while a 532-nm laser beam passes through its center.

$4.3 \mathrm{~mm} \times 0.2 \mathrm{~mm}$ ) grown via chemical vapor deposition (CVD) with a high NV density $\sim 10^{15} \mathrm{~cm}^{-3}$ (Apollo Diamond, Inc.) [Fig. 1(d)]. Four 650-nm long-pass optical filters (Edmund Optics NT62-979, cut into rectangular pieces with a water jet cutter) were placed in contact with the edges of the diamond. The filters transmitted most of the NV fluorescence band $(\sim 637-800 \mathrm{~nm})$, while reflecting scattered 532-nm light used to excite the NVs. Four chip-style Si photodiodes (Advanced Photonix PDB-C609-2) were affixed to the backs of the filters. Because the filters were on a 2-mm-thick quartz substrate, large active-area photodiodes $(6 \mathrm{~mm} \times 7 \mathrm{~mm})$ were employed to maximize the detection acceptance angle. Future designs may employ smaller detectors with thin optical filters, and fewer detectors if one or more edges of the diamond chip are polished and mirror coated.

We calibrated the prototype device's photon side-collection efficiency by comparing it against a microscope objective with known $\eta_{c}$, using a wide-field epifluorescence microscope configuration [Fig. 2(a)]. Light from a 532-nm laser was focused into the diamond sample by a microscope air objective $(\mathrm{NA}=0.40)$. The objective also collected NV fluorescence, and directed it to a filter and photodiode identical to those used for side collection. The objective's low NA guaranteed that its line of sight was not obstructed by the side-collection filters. In Fig. 2(d), we compare the integrated number of fluorescence photons detected with the two collection modalities during a 300-ns duration pulse of 532-nm excitation laser light, at various laser powers. The integrated photon count was measured by alternately connecting a charge-sensitive
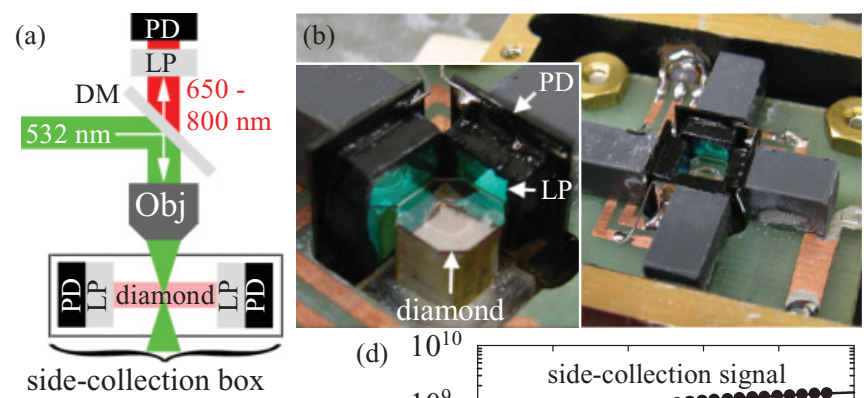

(c)
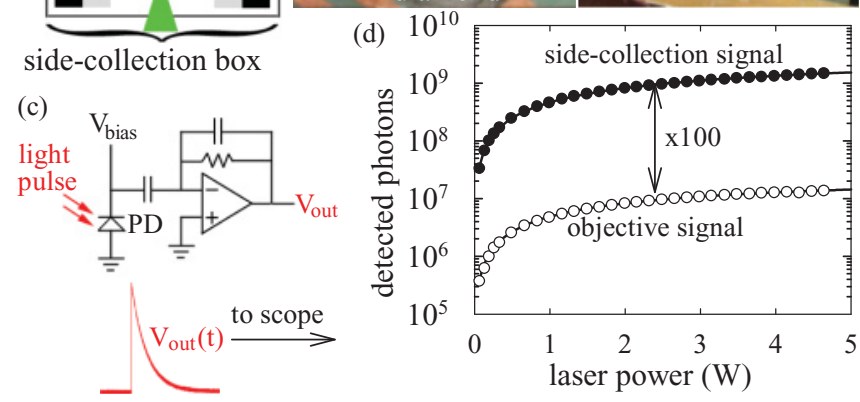

FIG. 2. (Color online) (a) Prototype device to compare the photon collection and detection efficiency of the side-collection technique with that of a 0.4-NA microscope objective (Obj), using a high NV-density diamond chip, 532-nm excitation laser, dichroic mirror (DM), 650-nm long-pass filter (LP), and Si photodiodes (PD). (b) Photo of the side-collection prototype device (partially disassembled for viewing of the interior). The inset shows the locations of the LPs and PDs in relation to the diamond chip. (c) Side-collection and microscope objective optical signals were recorded using a charge-sensitive amplifier. (d) NV fluorescence (number of photons) detected by the two collection modalities during a 300-ns pulse of the excitation laser $(532 \mathrm{~nm})$, as a function of laser power, shows a 100-times larger side-collection signal.

amplifier (Cremat Inc. CR-112) to the photodiode(s) of the two collection paths, and recording the average signal amplitude [Fig. 2(c)]. We found that the side-collection signal had a $100 \pm 5$ times larger photon count than the microscope objective signal under identical experimental conditions. The theoretically estimated collection efficiency for the microscope objective was $0.59 \% .{ }^{14,15}$ Transmission losses through the objective, dichroic, and filter reduced the fraction of light reaching the detector to $\eta_{c} \approx 0.47 \%$. The average quantum efficiency of the photodiode within the NV emission band at near-normal incidence $\approx 83 \%$, indicating $\eta_{d} \approx 0.39 \%$. This implies that the NV fluorescence detection efficiency of the side-collection prototype device $\approx 39 \%$, while the fraction of photons reaching the four side-collection photodiodes (over a wide range of incidence angles) was $\geqslant 47 \%$. Note that this is $\approx 5$ times larger than the ideal collection efficiency of a microscope objective with $\mathrm{NA}=1.49$, neglecting losses between the objective and detector. ${ }^{14,15}$

We also confirmed that the optical signals in the two detection paths were due to NV fluorescence by sweeping the frequency of a microwave field across the characteristic $\mathrm{NV}$ electron spin resonance at $2.87 \mathrm{GHz}$, with zero magnetic field. $^{21}$ The fluorescence signal in both detection paths decreased on resonance by a similar amount $\approx 17 \%$, indicating that the side-collection filters selected NV fluorescence as effectively as conventional fluorescence microscopy. 
(a)

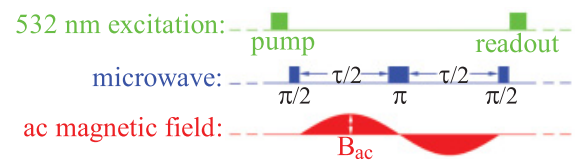

(b)

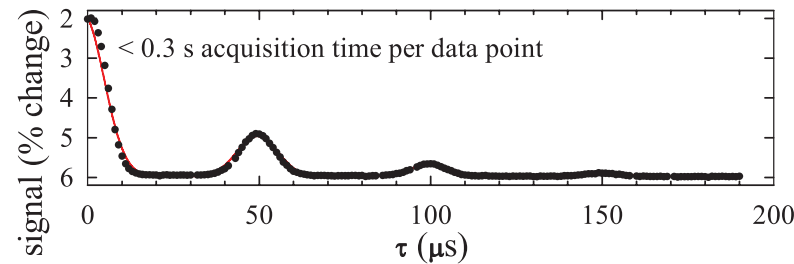

(c)

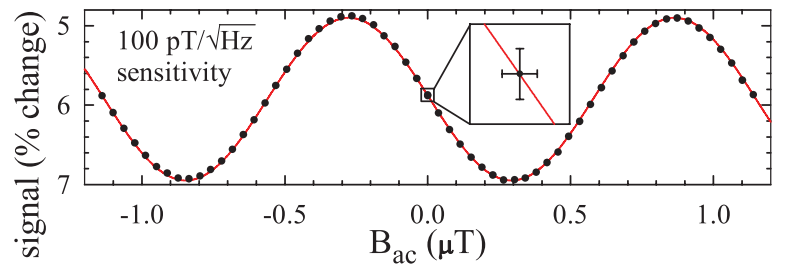

FIG. 3. (Color online) (a) Optical and microwave spin-echo pulse sequence used for NV-diamond ac magnetometry with the side-collection prototype device (Refs. 11 and 14). An optical pump pulse prepares the NV spins in the $\left|m_{s}=0\right\rangle$ state; a microwave spin-echo sequence allows the NV spins to probe an applied $20-\mathrm{kHz}$ magnetic field; and an optical readout pulse allows measurement of the change in NV spin-dependent fluorescence compared to the initial state. (b) By varying $\tau$ with $B_{\text {ac }}=0$, we measure the NV spin-echo decoherence curve shown, with a greatly enhanced signal-to-noise ratio (SNR) provided by the side-collection technique. (c) Varying $B_{\text {ac }}$ with $\tau=50 \mu$ s produces the NV-diamond ac magnetometry curve shown. The zoomed-in region of the curve near $B_{\mathrm{ac}}=0$ depicts the mean and standard deviation of a large number of ac magnetometry measurements, each lasting $57 \mu \mathrm{s}$. The $\pm 0.071 \%$ uncertainty in the signal (vertical error bars) corresponds to a $\pm 13 \mathrm{nT}$ uncertainty in $B_{\mathrm{ac}}$ (horizontal error bars) for a single measurement, yielding $100 \mathrm{pT} / \sqrt{\mathrm{Hz}}$ sensitivity for a laser excitation volume $\sim 10^{-4} \mathrm{~mm}^{3}$.

One important application of the side-collection technique is sensitive magnetometry using ensembles of NV spins in a diamond chip. ${ }^{13,22}$ To demonstrate this application using the side-collection prototype device, we performed ac magnetometry with $\sim 10^{8} \mathrm{NV}$ s contributing to the magnetometry signal in $\mathrm{a} \sim 10^{-4} \mathrm{~mm}^{3}$ laser excitation volume, using the standard spinecho technique described in Ref. 11 [Fig. 3(a)]. ${ }^{14}$ We applied a 37.5-G static magnetic field in the [111] direction, so that we could use an applied microwave field to resonantly select $\left|m_{s}=0\right\rangle \leftrightarrow\left|m_{s}=1\right\rangle$ spin transitions of [111]-oriented NVs, as in Ref. 13. (One-quarter of the total number of NV spins are resonantly manipulated with this method, as NV orientations are distributed equally among the four crystallographic axes.) By sweeping the spin-echo duration $(\tau)$, we generated a $T_{2}$ coherence curve with revivals at even multiples of the ${ }^{13} \mathrm{C}$ nuclear precession period [Fig. 3(b)]. ${ }^{11}$ We extracted $T_{2}=35 \mu$ s for this high NV-density diamond sample. We then generated the magnetometry curve in [Fig. 3(c)] by varying the amplitude of a $20-\mathrm{kHz}$ ac magnetic field, with $\tau=50 \mu \mathrm{s}$.

Magnetometer sensitivity $\eta$ is defined in terms of the minimum detectable magnetic field $B_{\min }$ after a given measurement averaging time $T, B_{\min } \equiv \eta / \sqrt{T}$. For NV ac magnetometers operating near the maximum slope of the ac magnetometry curve, $\eta$ can be written analytically in terms of the minimum detectable magnetic field $b_{\min }$ from a single measurement with duration $t_{m}$, assuming that $T / t_{m}$ measurements are made during an averaging interval $T$ :

$$
\eta=b_{\min } \sqrt{t_{m}} \simeq \frac{\pi \hbar}{4 \mu_{B}} \frac{\sqrt{t_{m}}}{(S / \sigma) \beta \tau},
$$

where $\mu_{B}$ is the Bohr magneton, $S / \sigma$ is the signal-to-noise ratio (SNR) of a single measurement, and $\beta$ is the measurement contrast (amplitude divided by mean of the ac magnetometry curve). ${ }^{22}$ For magnetometry measurements at zero ac field amplitude with $t_{m}=57 \mu \mathrm{s}$, we found $S / \sigma=1.4 \times 10^{3}$ [see Fig. 3(c)], corresponding to a sensitivity $\approx 100 \mathrm{pT} / \sqrt{\mathrm{Hz}} .3,23$ For an ideal measurement, $t_{m} \approx \tau \approx T_{2}$, and the SNR is limited by photon shot noise, $S / \sigma=\sqrt{N}$, where $N$ is the number of photons detected in a single measurement, so that $\eta \propto 1 /\left(\beta \sqrt{N T_{2}}\right)$. The best previously reported NV magnetometer sensitivity was $4 \mathrm{nT} / \sqrt{\mathrm{Hz}}$, using a single $\mathrm{NV}$ in an isotopically pure diamond with $T_{2}=1.8 \mathrm{~ms},{ }^{3}$ where typically $\beta \approx 0.15$ and $N \approx 0.024$ for such measurements. ${ }^{11}$ For our ac magnetometry measurement, $N \approx 2 \times 10^{8}$, illustrating the improved SNR that can be obtained by making ensemble measurements with enhanced collection efficiency. From Eq. (1) and the shot-noise-limited SNR $\left(\sqrt{N}=1.4 \times 10^{4}\right)$, we would have expected an ideal sensitivity $\approx 9 \mathrm{pT} / \sqrt{\mathrm{Hz}}$, but our magnetometer sensitivity was instead limited by electrical noise in the detection circuitry of the prototype side-collection device. ${ }^{14}$ We expect that this added noise can be significantly reduced in future, optimized side-collection instruments, and that shot-noise-limited sensitivities below $1 \mathrm{pT} / \sqrt{\mathrm{Hz}}$ could be achieved by increasing the measurement volume, and lengthening the NV $T_{2}$ through diamond engineering ${ }^{3}$ or dynamic decoupling techniques. ${ }^{24}$

The photon detection enhancement of the side-collection technique has many other potential applications. For example, magnetometry may be extended to magnetic field imaging by using a thin layer of NVs near the diamond chip surface ${ }^{13}$ by scanning the laser focus while making time-resolved side-collection fluorescence measurements. The high SNR provided by side collection also greatly increases the speed of NV ensemble measurements, which may be used to study decoherence processes and develop NV spin manipulation protocols for magnetometry and quantum information. For example, the NV decoherence and magnetometry data shown in Fig. 3 was generated with $<0.3 \mathrm{~s}$ of signal averaging time per data point, which is orders of magnitude shorter than the time that would be required to achieve comparable SNR using a microscope objective. The side-collection technique should also be applicable to single NV measurements using a tightly focused laser beam for NV excitation. However, because side collection accepts out-of-focus light, a thin, pure diamond would likely be required to isolate single NVs in the excitation volume. Using side collection to increase the photon count rate from a single NV could increase the speed and precision of NV super-resolution imaging ${ }^{9}$ and magnetometry. ${ }^{10}$ It would also improve NV quantum-state readout fidelity, and may be used in conjunction with existing techniques to achieve single-shot readout of the NV spin for quantum information applications. $^{7}$ 
We gratefully acknowledge the provision of diamond samples by Apollo Diamond and Element Six; and also informative discussions with Patrick Doering, David
Glenn, and Alexei Trifonov. This work was supported by NIST, NSF, and DARPA (QuEST and QuASAR programs). *rwalsworth@cfa.harvard.edu

${ }^{1}$ I. Aharonovich, S. Castelletto, D. A. Simpson, C.-H. Su, A. D. Greentree, and S. Prawer, Rep. Prog. Phys. 74, 076501 (2011).

${ }^{2}$ F. Jelezko, T. Gaebel, I. Popa, A. Gruber, and J. Wrachtrup, Phys. Rev. Lett. 92, 076401 (2004).

${ }^{3}$ G. Balasubramanian, P. Neumann, D. Twitchen, M. Markham, R. Kolesov, N. Mizuochi, J. Isoya, J. Achard, J. Beck, J. Tissler, V. Jacques, P. R. Hemmer, F. Jelezko, and J. Wrachtrup, Nat. Mater. 8, 383 (2009).

${ }^{4}$ A. Beveratos, R. Brouri, T. Gacoin, J.-P. Poizat, and P. Grangier, Phys. Rev. A 64, 061802 (2001).

${ }^{5}$ T. M. Babinec, B. J. M. Hausmann, M. Khan, Y. Zhang, J. R. Maze, P. R. Hemmer, and M. Loncar, Nat. Nanotechnol. 5, 195 (2010).

${ }^{6}$ M. V. G. Dutt, L. Childress, L. Jiang, E. Togan, J. Maze, F. Jelezko, A. S. Zibrov, P. R. Hemmer, and M. D. Lukin, Science 316, 1312 (2007).

${ }^{7}$ L. Jiang, J. S. Hodges, J. R. Maze, P. Maurer, J. M. Taylor, D. G. Cory, P. R. Hemmer, R. L. Walsworth, A. Yacoby, A. S. Zibrov, and M. D. Lukin, Science 326, 267 (2009).

${ }^{8}$ E. Togan, Y. Chu, A. S. Trifonov, L. Jiang, J. Maze, L. Childress, M. V. G. Dutt, A. S. Sorensen, P. R. Hemmer, A. S. Zibrov, and M. D. Lukin, Nature (London) 466, 730 (2010).

${ }^{9}$ E. Rittweger, K. Y. Han, S. E. Irvine, C. Eggeling, and S. W. Hell, Nat. Photonics 3, 144 (2009).

${ }^{10}$ P. C. Maurer, J. R. Maze, P. L. Stanwix, L. Jiang, A. V. Gorshkov, A. A. Zibrov, B. Harke, J. S. Hodges, A. S. Zibrov, A. Yacoby, D. Twitchen, S. W. Hell, R. L. Walsworth, and M. D. Lukin, Nat. Phys. 6, 912 (2010).

${ }^{11}$ J. R. Maze, P. L. Stanwix, J. S. Hodges, S. Hong, J. M. Taylor, P. Cappellaro, L. Jiang, M. V. G. Dutt, E. Togan, A. S. Zibrov, A. Yacoby, R. L. Walsworth, and M. D. Lukin, Nature (London) 455, 644 (2008).

${ }^{12}$ G. Balasubramanian, I. Y. Chan, R. Kolesov, M. Al-Hmoud, J. Tisler, C. Shin, C. Kim, A. Wojcik, P. R. Hemmer, A. Krueger, T. Hanke, A. Leitenstorfer, R. Bratschitsch, F. Jelezko, and J. Wrachtrup, Nature (London) 455, 648 (2008).
${ }^{13}$ L. M. Pham, D. Le Sage, P. L. Stanwix, T. K. Yeung, D. Glenn, A. Trifonov, P. Cappellaro, P. R. Hemmer, M. D. Lukin, H. Park, A. Yacoby, and R. L. Walsworth, New J. Phys. 13, 045021 (2011).

${ }^{14}$ See Supplemental Material at http://link.aps.org/supplemental/ 10.1103/PhysRevB.85.121202 for derivations of microscope objective collection efficiencies and the estimated side-collection efficiency, as well as additional information about the experimental methods used for ac magnetometry.

${ }^{15}$ R. J. Epstein, F. M. Mendoza, Y. K. Kato, and D. D. Awschalom, Nat. Phys. 1, 94 (2005).

${ }^{16}$ J. Tisler, G. Balasubramanian, B. Naydenov, R. Kolesov, B. Grotz, R. Reuter, J.-P. Boudou, P. A. Curmi, M. Sennour, A. Thorel, M. BoÌrsch, K. Aulenbacher, R. Erdmann, P. R. Hemmer, F. Jelezko, and J. Wrachtrup, ACS Nano 3, 1959 (2009).

${ }^{17}$ P. L. Stanwix, L. M. Pham, J. R. Maze, D. Le Sage, T. K. Yeung, P. Cappellaro, P. R. Hemmer, A. Yacoby, M. D. Lukin, and R. L. Walsworth, Phys. Rev. B 82, 201201 (2010).

${ }^{18}$ P. Siyushev, F. Kaiser, V. Jacques, I. Gerhardt, S. Bischof, H. Fedder, J. Dodson, M. Markham, D. Twitchen, F. Jelezko, and J. Wrachtrup, Appl. Phys. Lett. 97, 241902 (2010).

${ }^{19}$ J. P. Hadden, J. P. Harrison, A. C. Stanley-Clarke, L. Marseglia, Y.-L. D. Ho, B. R. Patton, J. L. O'Brien, and J. G. Rarity, Appl. Phys. Lett. 97, 241901 (2010).

${ }^{20}$ V. M. Acosta, E. Bauch, M. P. Ledbetter, C. Santori, K.-M. C. Fu, P. E. Barclay, R. G. Beausoleil, H. Linget, J. F. Roch, F. Treussart, S. Chemerisov, W. Gawlik, and D. Budker, Phys. Rev. B 80, 115202 (2009).

${ }^{21}$ A. Gruber, A. Dräbenstedt, C. Tietz, L. Fleury, J. Wrachtrup, and C. v. Borczyskowski, Science 276, 2012 (1997).

${ }^{22}$ J. Taylor, P. Cappellaro, L. Childress, L. Jiang, D. Budker, P. R. Hemmer, A. Yacoby, R. Walsworth, and M. D. Lukin, Nat. Phys. 4, 810 (2008).

${ }^{23}$ V. M. Acosta, E. Bauch, A. Jarmola, L. J. Zipp, M. P. Ledbetter, and D. Budker, Appl. Phys. Lett. 97, 174104 (2010).

${ }^{24}$ B. Naydenov, F. Dolde, L. T. Hall, C. Shin, H. Fedder, L. C. L. Hollenberg, F. Jelezko, and J. Wrachtrup, Phys. Rev. B 83, 081201 (2011). 